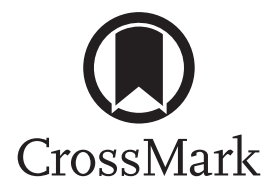

\title{
Bedaquiline: 10 years later, the drug susceptibility testing protocol is still pending
}

\author{
Max Salfinger ${ }^{1}$ and Giovanni Battista Migliori $^{2}$
}

Affiliations: ${ }^{1}$ Dept of Medicine, National Jewish Health, Denver, CO, USA. ${ }^{2}$ WHO Collaborating Centre for TB and Lung Diseases, Fondazione S. Maugeri, Care and Research Institute, Tradate, Italy.

Correspondence: Giovanni Battista Migliori, WHO Collaborating Centre for TB and Lung Diseases, Fondazione S. Maugeri, Care and Research Institute, via Roncaccio 16, 21049 Tradate, Italy.

E-mail: giovannibattista.miglioriafsm.it

@ERSpublications

Although bedaquiline is already used to treat MDR-TB neither DST nor MIC protocols are yet available to guide its use http://ow.ly/ESrRz

\section{Introduction}

With 9.0 million cases and 1.5 million deaths estimated in 2013, tuberculosis (TB) remains one of the world's deadliest communicable diseases; however, it is slowly declining each year and it is estimated that 37 million lives were saved between 2000 and 2013 through effective diagnosis and treatment [1]. Globally, 3.5\% of new and $20.5 \%$ of previously treated TB cases (480000 people) were estimated to have been multidrug-resistant (MDR)-TB in 2013, with $9.0 \%$ of them being affected by extensively drug-resistant (XDR)-TB.

If all notified TB patients (6.1 million new and previously treated) had been tested for drug resistance in 2013, an estimated 300000 cases of MDR-TB would have been detected, more than half of these being in India, China and the Russian Federation. In 2013, 136000 of the estimated 300000 MDR-TB patients who could have been detected were diagnosed and notified. This was equivalent to almost one in two (45\%), up from one in six in 2009. Progress in the detection of drug-resistant TB has been facilitated by the use of new, rapid molecular diagnostics $[1,2]$.

In the TB field, the therapeutic armamentarium is significant but the efficacy of the anti-TB drugs cannot be deemed equivalent: drugs that should be administered in cases of drug-resistant TB forms are less efficacious, are expensive and are more toxic $[3,4]$. From a clinical perspective, the main consequences are a poor prognosis (i.e. increased mortality), poor adherence because of the adverse events, and a longer duration of drug exposure linked to personal and social problems [5]

The World Health Organization (WHO)-recommended regimens include at least four drugs divided into five groups. Among them, pyrazinamide should be administered with a later-generation fluoroquinolone, ethionamide or prothionamide, cycloserine or para-aminosalicylic acid, and second-line injectable drug (i.e. amikacin, capreomycin or kanamycin). One of those drugs can be replaced by a group 5 drug in case of resistance (table 1). The conventional drug susceptibility testing (DST) for first- and second-line anti-TB drugs, performed in quality-assured laboratories, is required to tailor the treatment regimen, though this takes $>1$ month for definitive results. In the meantime, patients could be treated on the basis of the suggested WHO standardised regimens for the most prevalent epidemiological resistance pattern in the setting where the infection presumptively occurs and the resistance pattern of the index case [5]. The stepwise approach recommended by WHO to design an effective MDR-TB regimen is summarised in table 1 [6].

The poor efficacy of treatment and the challenges involved in the programmatic implementation of the current recommended MDR-TB regimen result in poor outcomes. A recent meta-analysis of outcomes for $>9000$ patients receiving treatment for pulmonary MDR-TB reported only a 54\% success rate; treatment

Received: Oct 282014 | Accepted: Nov 192014

Conflict of interest: None declared.

Copyright OERS 2015 


\section{TABLE 1 Stepwise approach to designing a multidrug-resistant (MDR) tuberculosis (TB) regimen}

\begin{tabular}{|c|c|c|c|}
\hline Step 1 & $\begin{array}{l}\text { Choose an injectable drug } \\
\text { from WHO group } 2\end{array}$ & $\begin{array}{l}\text { Kanamycin } \\
\text { Amikacin } \\
\text { Capreomycin }\end{array}$ & $\begin{array}{l}\text { Choose a drug based on DST and treatment history } \\
\text { Streptomycin is generally not used because of high rates of } \\
\text { resistance in patients with MDR-TB }\end{array}$ \\
\hline Step 2 & $\begin{array}{l}\text { Choose a later-generation } \\
\text { fluoroquinolone from } \\
\text { WHO group } 3\end{array}$ & $\begin{array}{l}\text { Levofloxacin } \\
\text { Moxifloxacin }\end{array}$ & $\begin{array}{c}\text { Use a later-generation fluoroquinolone } \\
\text { If levofloxacin (or ofloxacin) resistance is documented, } \\
\text { use moxifloxacin } \\
\text { Avoid moxifloxacin if possible when using bedaquiline }\end{array}$ \\
\hline Step 3 & Add WHO group 4 drugs & $\begin{array}{l}\text { Cycloserine/terizidone PAS } \\
\text { Ethionamide/prothionamide }\end{array}$ & $\begin{array}{c}\text { Add two or more group } 4 \text { drugs until there are at least four } \\
\text { second-line anti-TB drugs likely to be effective } \\
\text { Ethionamide/prothionamide is considered the most effective } \\
\text { Group } 4 \text { drug } \\
\text { Consider treatment history, side-effect profile and cost } \\
\text { DST is not considered reliable for the drugs in this group }\end{array}$ \\
\hline Step 4 & Add WHO group 1 drugs & $\begin{array}{l}\text { Pyrazinamide } \\
\text { Ethambutol }\end{array}$ & $\begin{array}{l}\text { Pyrazinamide is routinely added in most regimens } \\
\text { Ethambutol can be added if the criteria for an effective } \\
\text { drug are met } \\
\text { If isoniazid is unknown or pending, it can be added to the } \\
\text { regimen until DST results become available }\end{array}$ \\
\hline Step 5 & Add WHO group 5 drugs & $\begin{array}{c}\text { Bedaquiline } \\
\text { Linezolid } \\
\text { Clofazimine } \\
\text { Amoxicillin/clavulanate } \\
\text { Imipenem/cilastatin plus } \\
\text { clavulanate } \\
\text { Meropenem plus clavulanate } \\
\text { High-dose isoniazid } \\
\text { Clarithromycin } \\
\text { Thioacetazone }\end{array}$ & $\begin{array}{l}\text { Consider adding group } 5 \text { drugs if four second-line anti-TB } \\
\text { drugs are not likely to be effective from WHO groups } 2-4 \\
\text { If drugs are needed from this group, ensure to use two or } \\
\text { more of them } \\
\text { DST is not standardised for the drugs in this group }\end{array}$ \\
\hline
\end{tabular}

WHO: World Health Organization; DST: drug susceptibility testing; PAS: para-aminosalicylic acid. Reproduced and modified from [6] with permission from the publisher.

default, mortality and treatment failure rates were $23 \%, 15 \%$ and $8 \%$, respectively [7]. Treatment success in XDR-TB cases was even worse $(40 \%, 16 \%, 15 \%$ and $22 \%$, respectively) [8,9]. Analysis of outcomes from TB programmes operated by Médecins Sans Frontières showed similar results, with an overall treatment success rate of 55\% for MDR-TB and only $13 \%$ for XDR-TB [10].

\section{Bedaquiline}

Bedaquiline (also known as TMC207 and R207910) is the lead compound of a series of recently discovered diarylquinolines and was described in a landmark article in Science in 2005 [11]. As it inhibits a new target, it is suggested that bedaquiline is active against both drug-susceptible and drug-resistant isolates of Mycobacterium tuberculosis. Bedaquiline has a unique spectrum of potent and selective antimycobacterial activity in vitro. The range of minimal inhibitory concentrations (MICs) for the international reference strain $M$. tuberculosis $\mathrm{H} 37 \mathrm{Rv}$ and six fully antibiotic-susceptible isolates was 0.030 $0.120 \mu \mathrm{g} \cdot \mathrm{mL}^{-1}$ versus $0.500 \mu \mathrm{g} \cdot \mathrm{mL}^{-1}$ for rifampicin and $0.120 \mu \mathrm{g} \cdot \mathrm{mL}^{-1}$ for isoniazid. Bedaquiline demonstrated similar in vitro efficacy against $M$. tuberculosis clinical isolates resistant to the anti-TB drugs isoniazid, rifampicin, streptomycin, ethambutol, pyrazinamide and moxifloxacin. Bedaquiline did not inhibit purified $M$. tuberculosis DNA gyrase, the target of quinolones [11]. Indeed, using the BACTEC culture system (BD, Franklin Lakes, NJ, USA), inhibition of bacterial growth was observed when MDR-TB strains were exposed to fixed concentrations of bedaquiline. 30 isolates of MDR-TB tested were susceptible to bedaquiline at $0.100 \mu \mathrm{g} \cdot \mathrm{mL}^{-1}$; of these, $17(57 \%)$ were susceptible to $\mathrm{R} 207910$ at $0.010 \mu \mathrm{g} \cdot \mathrm{mL}^{-1}$. Using the same method, similar susceptibility among 10 fully antibiotic-susceptible strains was observed [11].

Complementation studies have verified that a mutant atpE gene is responsible for resistance to bedaquiline, implying that the AtpE gene product is the target of bedaquiline in mycobacteria [11]. The atpE gene encodes the subunit $\mathrm{c}$ of the ATP synthase of $M$. tuberculosis and has been sequenced from in vitro-selected mutants resistant to the drug, identifying two mutations (A63P and I66M) [12].

On December 28, 2012, on the basis of data from two phase IIb trials (i.e. well-controlled trials to evaluate the efficacy and safety of drugs in patients with a disease or condition to be treated, diagnosed or prevented), the US Food and Drug Administration approved the use of bedaquiline under the provisions of the accelerated approval regulations for "serious or life-threatening illnesses" $[13,14]$. 
No cross-resistance was found between bedaquiline and isoniazid, rifampicin, streptomycin, ethambutol, pyrazinamide, amikacin or moxifloxacin. A four-fold increase in bedaquiline MIC values (suggesting acquired resistance) has been observed in 13 out of 28 patients with paired M. tuberculosis (baseline and post-baseline) isolates during clinical studies; 10 out of 13 patients had isolates with matching genotypes, which is evidence against reinfection with a new M. tuberculosis strain, and of these, nine had evidence of treatment failure or relapse. In vitro studies have revealed that bedaquiline had a bacteriostatic effect at low serum levels $\left(0.3 \mu \mathrm{g} \cdot \mathrm{mL}^{-1}\right)$ that might predispose to development of acquired resistance [14].

Similar to second-line drugs used for the treatment of MDR-TB, bedaquiline use in the treatment of pulmonary MDR-TB should be accompanied by microbiological monitoring with one sputum specimen submitted for culture monthly throughout and at the end of treatment, even after conversion to negative culture, which is consistent with the standard approach to treatment and care of patients with MDR-TB in the USA. Any monthly specimen that grows M. tuberculosis, including one before treatment initiation with bedaquiline, should be referred to a laboratory for surveillance of bedaquiline resistance [14].

The WHO Expert Group concluded that the randomised, double-blind, design of the pivotal study was of high quality, although information on the desired sample size and on the actual randomisation process was not available. The WHO Expert Group was, however, concerned about the use of modified intention-to-treat analysis (and subsequent assumptions made), as well as the representativeness of the study population. The WHO Expert Group was also concerned about the low cure rate at 120 weeks observed in the placebo group, when compared with those reported from recent published reviews. This could indicate that the patients included in the trial were not representative of the MDR-TB population at large and that the effects observed in the bedaquiline arm may not be reproducible under programme conditions. Concern was also expressed that, in the absence of patient data on DST status in the different arms, the background regimen used in various sites of the trial may not have been compliant with WHO recommendations [15].

The WHO Expert Group recommended that: 1) in the absence of a specific DST, resistance to bedaquiline should be monitored through assessment of MICs; and 2) resistance to other anti-TB drugs should be monitored following WHO recommendations [15].

Novel drugs currently in phase II or later clinical trials are derived from four classes of compounds: nitroimidazoles, diarylquinolines, oxazolidinones and diamines. Bedaquiline has been recommended for use in adults with pulmonary MDR-TB by WHO. Delamanid (Deltyba; Otsuka Novel Products GmbH, Munich, Germany) was approved by the European Medicines Agency on April 28, 2014 and by the Pharmaceutical and Medical Devices Agency in Japan on July 4, 2014. Otsuka has filed a new drug application (NDA) in Korea and hopes to file an NDA in the USA soon. There is, therefore, real potential that two new drugs will be available for the treatment of MDR-TB in the near future (table 2). In addition, existing drugs not yet licensed for the treatment of MDR-TB, such as linezolid [16], clofazimine, moxifloxacin and other drugs at an earlier stage in the drug development pipeline (e.g. PA-824 and sutezolid), have shown promising results (table 2) [10, 17].

\section{New discoveries: cross resistance between clofazimine and bedaquiline}

Recently, the anti-leprosy drug clofazimine has also become of renewed interest for the treatment of MDR-TB. To understand possible resistance mechanisms, clofazimine-resistant M. tuberculosis mutants were isolated in vitro by HARTKOORN et al. [18] and, unexpectedly, found to be cross-resistant to bedaquiline. Mutations in the transcriptional regulator gene Rv0678 (a transcriptional repressor of the genes encoding the MmpS5-MmpL5 efflux pump) with concomitant upregulation of the multisubstrate efflux pump MmpL5 accounted for this cross-resistance. Mutation in Rv0678 should therefore be considered a confounding factor for the treatment of TB with clofazimine or bedaquiline.

In the absence of data explaining non-atpE-mediated bedaquiline resistance, it remains to be determined whether mutations in Rv0678 are involved and whether these mutants display cross-resistance to clofazimine. The Global Alliance for TB Drug Development is currently conducting clinical trials to evaluate the activity of both clofazimine and bedaquiline in combination regiments for the treatment of TB. To date, very little is known about the potential sources of drug resistance in such regimens; however, mutations in Rv0678 should be taken into consideration as a possible confounding factor [18].

ANDRIES et al. [19] reported that non-target-based resistance to bedaquiline and cross-resistance to clofazimine are due to mutations in Rv0678. Efflux-based resistance was identified in paired isolates from patients treated with bedaquiline as well as in mice, in which it was confirmed to decrease bactericidal efficacy. The efflux inhibitors verapamil and reserpine decreased the MIC of bedaquiline and clofazimine in vitro, but verapamil failed to increase the bactericidal effect of bedaquiline in mice and was unable to reverse efflux-based resistance in vivo. Cross-resistance between bedaquiline and clofazimine may have important clinical implications as both drugs are accessible for clinical use. 
TABLE 2 Summary of the key drugs used for the treatment of multidrug-/extensively drug-resistant tuberculosis (TB)

Chemical class Mechanism of action Details

\section{New drugs}

Bedaquiline (TMC-207)

Delamanid (OPC-67683)

\author{
Sutezolid (PNU-100480)
}

AZD-5847

PA-824

SQ-109

Drugs originally developed with different indications than TB Clofazimine

Linezolid

Moxifloxacin
Nitroimidazole

Inhibits mycolic acid cell wall

Oxazolidinone

Oxazolidinone

Nitroimidazole

Oxazolidinone
Fluoroquinolone
Inhibits mycobacterial ATP synthase

Inhibits microbial protein synthesis by blocking 505 ribosomal subunit

Inhibits microbial protein synthesis by blocking $50 \mathrm{~S}$ ribosomal subunit Inhibits cell wall synthesis Inhibits cell wall synthesis

Riminophenazine

Binds to mycobacterial DNA (disrupting the cell cycle) and to bacterial potassium transporters, thereby inhibiting their function

Inhibits microbial protein synthesis by blocking $50 \mathrm{~S}$ ribosomal subunit Inhibits DNA gyrase
Clinical development (phase III) Approved by US Food and Drug Administration and European Medicines Agency

Included in WHO group 5 drugs Clinical development (phase III) Approved by European Medicines Agency

Included in WHO Group 5 drugs

Clinical development (phase II)

Clinical development (phase II)

Clinical development (phase IIb) Clinical development (phase II)

Clinical development (phase II) Included in WHO Group 5 drugs

Clinical development (phase II) Included in WHO Group 5 drugs Clinical development (phase III) Included in WHO Group 3 drugs

WHO: World Health Organization.

The patient described in a letter in this issue of the European Respiratory Journal (ERJ) indicates that emergence of drug resistance to bedaquiline is an already ongoing threat: SomosKovi et al. [20] provide the first non-trial in vivo evidence of acquired resistance due to a mutation in Rv0678, and its association with clofazimine and bedaquiline cross-resistance in an M. tuberculosis isolate from a patient with MDR-TB. In January 2011, a Tibetan refugee was diagnosed with a bilateral and radiologically advanced MDR-TB at the Swiss Reference Center for Mycobacteria (Zurich, Switzerland). The M. tuberculosis isolate from the patient showed resistance to isoniazid, rifampicin, pyrazinamide, ethionamide, linezolid, moxifloxacin and streptomycin by quantitative DST in the MGIT 960 system (BD).

In order to strengthen the efficacy of therapy with the less potent second-line drugs, the patient received bedaquiline on a compassionate basis between September 2011 and February 2012. Culture conversion was confirmed at the end of October 2011. The patient remained culture negative and therapy was terminated in March 2013. In August 2013, the patient was re-admitted with fever, cough and acid-fast bacillus-positive sputum microscopy. Therapy was re-initiated with cycloserine, capreomycin, para-aminosalicylic acid, ethambutol, clofazimine and inhaled amikacin. Re-application for bedaquiline treatment was rejected by the manufacturer on the basis that the patient had already received treatment on a compassionate basis for 6 months. DST of the relapse isolate in 2013 confirmed the previous resistance pattern but surprisingly revealed additional resistance to clofazimine. The 2011 isolate was susceptible to clofazimine. Most notably, the patient never received clofazimine. Genotyping using 24-locus mycobacterial interspersed repetitive unit-variable-number tandem repeats did not identify differences between the post-relapse and the previous isolates from 2011. Analysis of the Rv0678 gene of the initial isolate of the patient from 2011 showed a wild-type sequence. In contrast, the Rv0678 gene sequence of the August 2013 isolate revealed a mutation at nucleotide position 2 (GTG $\rightarrow$ GCG) resulting in the loss of the start codon (replacement of methionine by alanine), suggesting that overexpression of the mmpL5 efflux pump gene due to impaired Rv0678 function is responsible for the observed resistance. Notably, the relapse isolates of the patient did not have any mutations in the atpE gene [20].

The threat of emerging bedaquiline resistance associated with this cross-resistance mechanism is corroborated by a recent publication on the compassionate use of bedaquiline in two patients with XDR-TB and pre-XDR-TB who received clofazimine therapy as part of their combination therapy [21]. 


\section{Conclusions}

The article in this issue of the ERJ [20] has several elements of interest. First, the scientific community was surprised to learn about cross-resistance between clofazimine and bedaquiline, here presented for the first time in a patient in clinical practice. Second, in a situation with a real risk of developing drug resistance, a balance needs to be found between easy access to the drug for patients (at a reasonable price) and rational use of the drug within properly designed background regimens [22]. Third, the scientific community needs to share all existing pieces of information deriving from the use of this new drug in order to have better information on its safety and efficacy. Finally, bedaquiline needs to be more widely accessible in order to evaluate best protocols for DST, MIC analyses and protocols for therapeutic drug monitoring [23-25]. In the original 2005 Science article, DST was performed using the radiometric BACTEC 460TB, which has since been discontinued. Now, 10 years later, there is still no published protocol for DST and MIC determination for bedaquiline.

As the new WHO End TB Strategy calls for TB pre-elimination and elimination (defined as $<10$ TB cases and one TB case per million population, respectively), national TB programmes need new drugs to be used, in rational manner, within new short, effective and safe regimens [1, 26, 27].

\section{References}

1 World Health Organization. Global tuberculosis report 2014. Document WHO/HTM/TB/2014.08. Geneva, World Health Organization, 2014.

2 Weyer K, Mirzayev F, Migliori GB, et al. Rapid molecular TB diagnosis: evidence, policy making and global implementation of Xpert MTB/RIF. Eur Respir J 2013; 42: 252-271.

3 Diel R, Rutz S, Castell S, et al. Tuberculosis: cost of illness in Germany. Eur Respir J 2012; 40: 143-151.

4 Diel R, Vandeputte J, de Vries G, et al. Costs of tuberculosis disease in the European Union: a systematic analysis and cost calculation. Eur Respir J 2014; 43: 554-565.

5 Sotgiu G, Migliori GB. Facing multi-drug resistant tuberculosis. Pulm Pharmacol Ther 2014 [In press DOI: 10.1016/j.pupt.2014.04.006].

6 World Health Organization. Companion handbook to the WHO guidelines for the programmatic management of drug-resistant tuberculosis. Document WHO/HTM/TB/2014.11. Geneva, World Health Organization, 2014.

7 Ahuja SD, Ashkin D, Avendano M, et al. Multidrug resistant pulmonary tuberculosis treatment regimens and patient outcomes: an individual patient data meta-analysis of 9,153 patients. PLoS Med 2012; 9: e1001300.

8 Falzon D, Gandhi N, Migliori GB, et al. Resistance to fluoroquinolones and second-line injectable drugs: impact on MDR-TB outcomes. Eur Respir J 2013; 42: 156-168.

9 Migliori GB, Sotgiu G, Gandhi NR, et al. Drug resistance beyond extensively drug-resistant tuberculosis: individual patient data meta-analysis. Eur Respir J 2013; 42: 169-179.

10 Brigden G, Nyang'wa BT, du Cros P, et al. Principles for designing future regimens for multidrug-resistant tuberculosis. Bull World Health Organ 2014; 92: 68-74.

11 Andries K, Verhasselt P, Guillemont J, et al. A diarylquinoline drug active on the ATP synthase of Mycobacterium tuberculosis. Science 2005; 307: 223-227.

12 Petrella S, Cambau E, Chauffeur A, et al. Genetic basis for natural and acquired resistance to the diarylquinoline R207910 in mycobacteria. Antimicrob Agents Chemother 2006; 50: 2853-2856.

13 Cox EM. FDA accelerated approval letter to Janssen Research and Development (21CFR314.500). www.accessdata. fda.gov/drugsatfda_docs/appletter/2012/204384Orig1s000ltr.pdf

14 Centers for Disease Control and Prevention. Provisional CDC Guidelines for the use and safety monitoring of bedaquiline fumarate (Sirturo) for the treatment of multidrug-resistant tuberculosis. MMWR 2013; 62: 1-12.

15 World Health Organization. The use of bedaquiline in the treatment of multidrug-resistant tuberculosis: interim policy guidance. Document WHO/HTM/TB/2013.6. Geneva, World Health Organization, 2013.

16 Sotgiu G, Centis R, D'Ambrosio L, et al. Efficacy, safety and tolerability of linezolid containing regimens in treating MDR-TB and XDR-TB: systematic review and meta-analysis. Eur Respir J 2012; 40: 1430-1442.

17 Migliori GB, Sotgiu G. Treatment of tuberculosis: have we turned the corner? Lancet 2012; 380: 955-957.

18 Hartkoorn RC, Uplekar S, Cole ST. Cross-resistance between clofazimine and bedaquiline through upregulation of MmpL5 in Mycobacterium tuberculosis. Antimicrob Agents Chemother 2014; 58: 2979-2981.

19 Andries K, Villellas C, Coeck N, et al. Acquired resistance of Mycobacterium tuberculosis to bedaquiline. PLoS One 2014; 9: e102135.

20 Somoskovi A, Bruderer V, Hömke R, et al. A mutation associated with clofazimine and bedaquiline cross-resistance in MDR-TB following bedaquiline treatment. Eur Respir J 2015; 45: 554-557.

21 Tiberi S, De Lorenzo S, Centis R, et al. Bedaquiline in MDR/XDR-TB cases: first experience on compassionate use. Eur Respir J 2014; 43: 289-292.

22 Migliori GB, Lienhardt C, Weyer K, et al. Ensuring rational introduction and responsible use of new TB tools: outcome of an ERS multisector consultation. Eur Respir J 2014; 44: 1412-1417.

23 Srivastava S, Peloquin CA, Sotgiu G, et al. Therapeutic drug management: is it the future of multidrug-resistant tuberculosis treatment? Eur Respir J 2013; 42: 1449-1453.

24 Bolhuis MS, van Altena R, van Soolingen D, et al. Clarithromycin increases linezolid exposure in multidrug-resistant tuberculosis patients. Eur Respir J 2013; 42: 1614-1621.

25 Akkerman OW, van Altena R, Klinkenberg T, et al. Drug concentration in lung tissue in multidrug-resistant tuberculosis. Eur Respir J 2013; 42: 1750-1752.

26 Diel R, Loddenkemper R, Zellweger JP, et al. Old ideas to innovate tuberculosis control: preventive treatment to achieve elimination. Eur Respir J 2013; 42: 785-801.

27 D'Ambrosio L, Dara M, Tadolini M, et al. Tuberculosis elimination: theory and practice in Europe. Eur Respir J 2014; 43: 1410-1420. 